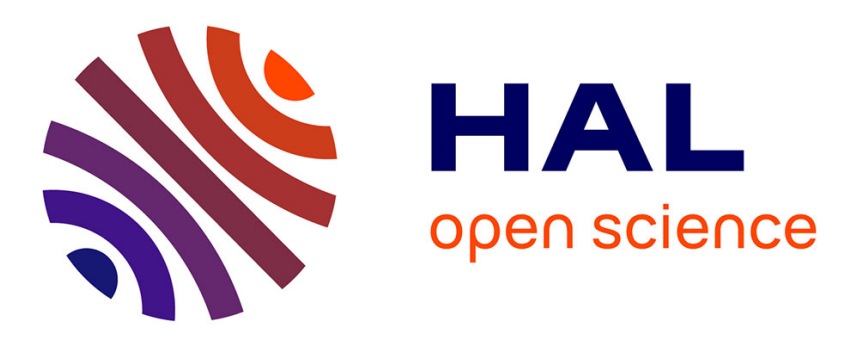

\title{
Face-based Smoothed Finite Element Method for Real-time Simulation of soft tissue
}

Andrea Mendizabal, Rémi Bessard Duparc, Huu Phuoc Bui, Christoph J Paulus, Igor Peterlik, Stéphane Cotin

\section{To cite this version:}

Andrea Mendizabal, Rémi Bessard Duparc, Huu Phuoc Bui, Christoph J Paulus, Igor Peterlik, et al.. Face-based Smoothed Finite Element Method for Real-time Simulation of soft tissue. SPIE Medical Imaging, Feb 2017, Orlando, United States. hal-01444595

\section{HAL Id: hal-01444595 \\ https://inria.hal.science/hal-01444595}

Submitted on 24 Jan 2017

HAL is a multi-disciplinary open access archive for the deposit and dissemination of scientific research documents, whether they are published or not. The documents may come from teaching and research institutions in France or abroad, or from public or private research centers.
L'archive ouverte pluridisciplinaire HAL, est destinée au dépôt et à la diffusion de documents scientifiques de niveau recherche, publiés ou non, émanant des établissements d'enseignement et de recherche français ou étrangers, des laboratoires publics ou privés. 


\title{
Face-based Smoothed Finite Element Method for Real-time Simulation of soft tissue
}

\author{
Andrea Mendizabal ${ }^{\mathrm{a}}$, Rémi Bessard Duparc ${ }^{\mathrm{a}}$, Huu Phuoc Bui ${ }^{\mathrm{b}}$, Christoph J. Paulus ${ }^{\mathrm{a}}$, Igor \\ Peterlik $^{\mathrm{a}}$, and Stéphane Cotin ${ }^{\mathrm{a}}$ \\ ${ }^{a}$ Inria Nancy Grand Est, 54603 Villers-les-Nancy, France \\ ${ }^{\mathrm{b}}$ University of Strasbourg, CNRS, ICube, F-67000 Strasbourg, France
}

\begin{abstract}
In soft tissue surgery, a tumor and other anatomical structures are usually located using the preoperative CT or MR images. However, due to the deformation of the concerned tissues, this information suffers from inaccuracy when employed directly during the surgery. In order to account for these deformations in the planning process, the use of a bio-mechanical model of the tissues is needed. Such models are often designed using the finite element method (FEM), which is, however, computationally expensive, in particular when a high accuracy of the simulation is required.

In our work, we propose to use a smoothed finite element method (S-FEM) in the context of modeling of the soft tissue deformation. This numerical technique has been introduced recently to overcome the overly stiff behavior of the standard FEM and to improve the solution accuracy and the convergence rate in solid mechanics problems. In this paper, a face-based smoothed finite element method (FS-FEM) using 4-node tetrahedral elements is presented. We show that in some cases, the method allows for reducing the number of degrees of freedom, while preserving the accuracy of the discretization. The method is evaluated on a simulation of a cantilever beam loaded at the free end and on a simulation of a 3D cube under traction and compression forces. Further, it is applied to the simulation of the brain shift and of the kidney's deformation. The results demonstrate that the method outperforms the standard FEM in a bending scenario and that has similar accuracy as the standard FEM in the simulations of the brain-shift and of the kidney's deformation.
\end{abstract}

Keywords: smoothed finite element method, strain smoothing, real-time simulation, soft tissue modeling

\section{DESCRIPTION OF PURPOSE}

In medical simulations, real-time performance is a strong requirement for clinical use. The FS-FEM was proposed by Liu et $a l^{1}$ in order to improve the accuracy of the FE-based modeling. Recent works have adapted the FSFEM to biological research. ${ }^{2}$ However, to our best knowledge, the method has not been studied or adapted in the context of real-time simulations and no implementation of the S-FEM approach allowing for real-time computation exists.

In this paper, we employ the FS-FEM approach in the context of navigation during the brain tumor surgery ${ }^{3-5}$ and partial nephrectomy. During the intervention, the position of the tumor has to be accurately estimated from the pre-operative CT or MR data. In the case of brain surgery, a burr hole is drilled in the skull in order to insert surgical instruments and the cerebrospinal fluid (CSF) leaks resulting in the motion and deformation of the brain known as the brain shift. As it is difficult to predict the exact amount of the brain shift before the leak of the CSF, the pre-operative planning of the intervention becomes inaccurate ${ }^{6,7}$ Hence, it is necessary to design a novel technique combining the pre-operative data with intra-operative tracking which would be used to drive a simulation based on an accurate biomechanical model. Such technique would dramatically improve the accuracy of navigation, thus reducing the risk to the patient. The partial removal of a kidney is a complex procedure that requires a highly experienced surgeon to be successful. A consequence of this is that several surgeons prefer doing a total nephrectomy in order to reduce the risk of failure. Hence, it is important to design

Corresponding author: Stéphane Cotin, e-mail: stephane.cotin@inria.fr 
a technique that predicts accurately the tumor location intra-operatively in order to remove as less as possible healthy kidney tissue and preserve its function.

In this work, we show that FS-FEM has similar accuracy as the standard FEM in the simulations of the brain-shift and of the kidney's deformation, while it outperforms the standard FEM in a bending beam scenario.

\section{METHOD}

A three-dimensional computational domain $\Omega$ is divided into $n_{e}$ tetrahedral elements. Over each element the displacement field is defined as follows

$$
\widetilde{\boldsymbol{u}}(x)=\sum_{I=1}^{N_{d}} \mathbf{N}_{I} \mathbf{u}_{I}
$$

and the compatible strain is given by

$$
\widetilde{\boldsymbol{\epsilon}}(x)=\sum_{I=1}^{N_{d}} \mathbf{B}_{I} \mathbf{u}_{I} .
$$

$\mathbf{N}_{I}$ is the shape function at node $I, N_{d}$ is the total number of independent nodal basis shape functions, and $\mathbf{B}_{I}$ is the strain displacement matrix at node I which size is $(6 \times 3)$. $\widetilde{\boldsymbol{u}}$ and $\widetilde{\boldsymbol{\epsilon}}$ are the displacement and the strain computed using the standard FEM. The S-FEM smooths the strain $\widetilde{\boldsymbol{\epsilon}}$ over the boundaries of the elements using the so-called smoothing domains. For the face-based smoothed finite element method, these domains are associated to the faces of the elements and are obtained by connecting portions of elements sharing a face $k$ (see Fig. 1 left). They are constructed on top of the element mesh, as a non overlapping and no gap subdivision of $\Omega$ such that:

$$
\Omega=\cup_{k=1}^{n_{s}} \Omega_{k}^{s}
$$

where $n_{s}$ is the total number of smoothing domains. In our case, the number of smoothing domains is equal to the number of faces i.e. $n_{s}=n_{f}$ where $n_{f}$ is the number of faces.
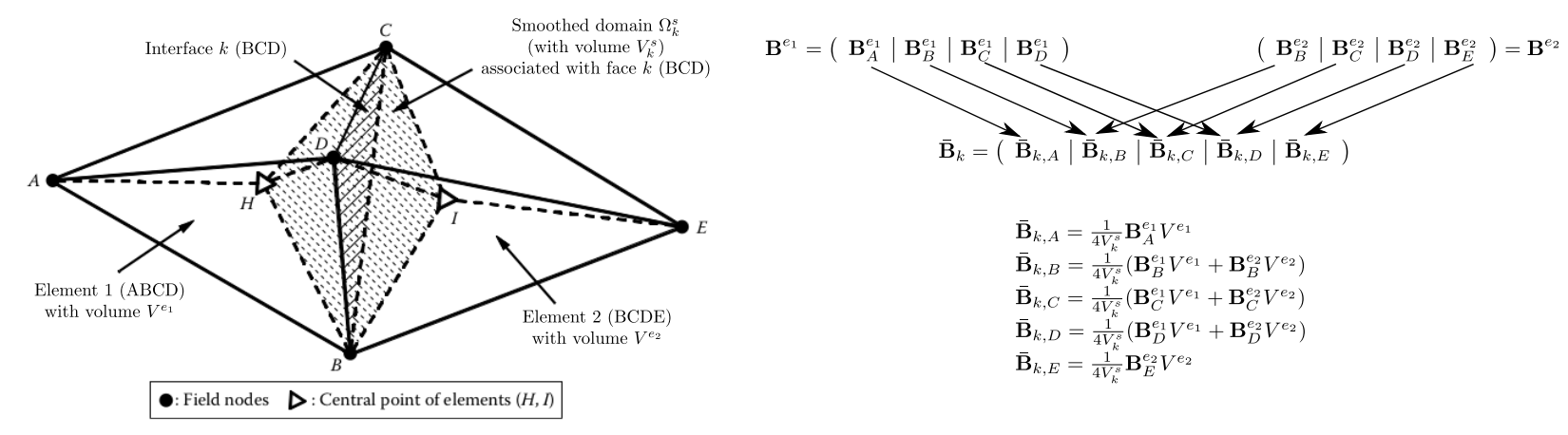

Figure 1. Left: Two adjacent tetrahedral elements and the smoothing domain (shaded) formed based on their interface $\mathrm{k}$ in FS-FEM. Right: Example of the assembling process of the smoothed strain displacement matrix.

The smoothed strain $\overline{\boldsymbol{\epsilon}}$ is obtained through a spatial average of the standard strain $\widetilde{\boldsymbol{\epsilon}}$ over the smoothing domains :

$$
\overline{\boldsymbol{\epsilon}}\left(x_{k}\right)=\int_{\Omega_{k}^{s}} \tilde{\boldsymbol{\epsilon}}(x) \hat{W}\left(x_{k}-x\right) d \Omega .
$$

$\hat{W}\left(x_{k}-x\right)$ is the smoothing (or weight) function, that is compactly supported, positive, centered at $x_{k}$ and verifying $\int_{\Omega_{k}^{s}} \hat{W}\left(x_{k}-x\right) d \Omega=1$. The most common expression for it is given by :

$$
\hat{W}\left(x_{k}-x\right)= \begin{cases}\frac{1}{V_{k}^{s}} & \text { if } x \in \Omega_{k}^{s} \cup \Gamma_{k}^{s} \\ 0 & \text { elsewhere. }\end{cases}
$$


$V_{k}^{s}$ is the volume of the smoothing domain $\Omega_{k}^{s}$ that is obtained by $V_{k}^{s}=\frac{1}{4} \sum_{m=1}^{n_{k}^{e}} V^{e_{m}}$. In this formula, $V^{e_{m}}$ is the volume of the $\mathrm{m}$-th element associated to face $\mathrm{k}$ and $n_{k}^{e}$ is the number of elements associated to face $\mathrm{k}$ (e.g. $n_{k}^{e}=1$ for a boundary face and $n_{k}^{e}=2$ for an inner face). Plugging (4) into (3), the smoothed strain field obtained is :

$$
\overline{\boldsymbol{\epsilon}}\left(x_{k}\right)=\overline{\boldsymbol{\epsilon}}=\frac{1}{V_{k}^{s}} \int_{\Omega_{k}^{s}} \tilde{\boldsymbol{\epsilon}}(x) d \Omega .
$$

In other words, the smoothed strain displacement matrix linked to face $k$ weights and averages the strain displacement matrix $\mathbf{B}^{e_{m}}$ of the two elements sharing the face $k$ (e.g. $m=1$ and $m=2$ ). In the case of a tetrahedral mesh, the smoothing is performed during the local assembling process (see Fig. 1 right for details) which is expressed as

$$
\overline{\mathbf{B}}_{k}=\frac{1}{V_{k}^{s}} \sum_{m=1}^{n_{k}^{e}} \frac{1}{4} V^{e_{m}} \mathbf{B}^{e_{m}} .
$$

As a result of the assembling process, $\overline{\mathbf{B}}_{k}$ is a $(6 \times 12)$ matrix for a boundary face and a $(6 \times 15)$ matrix for an inner face. The entries of $\overline{\mathbf{B}}_{k}$ are constant over the smoothing domain $\Omega_{k}^{s}$.

For linear tetrahedral elements, the strain is constant over the smoothing domains $\Omega_{k}^{s}$ and the local smoothed stiffness matrix $\overline{\mathbf{K}}_{k}$ is given by :

$$
\overline{\mathbf{K}}_{k}=\int_{\Omega_{k}^{s}} \overline{\mathbf{B}}_{k}^{T} \mathbf{C} \overline{\mathbf{B}}_{k} d \Omega=\overline{\mathbf{B}}_{k}^{T} \mathbf{C} \overline{\mathbf{B}}_{k} V_{k}^{s} .
$$

with the standard constitutive matrix $\mathbf{C}$ for linear elasticity. For an inner face, $\overline{\mathbf{K}}_{k}$ is a $(15 \times 15)$ matrix, while it is a $(12 \times 12)$ matrix (same as in standard FEM) for a boundary face.

Once the local matrix is computed, the global assembly differs from that of the standard FEM since on the local level, the matrix sizes are different which results in an increased density of the system matrix on the global level. The global smoothed stiffness matrix $\overline{\mathbf{K}}$ is computed by an assembling process as :

$$
\overline{\mathbf{K}}=\sum_{k=1}^{n_{s}} \overline{\mathbf{K}}_{k}
$$

Since we are in the linear case, this assembling can be done at the first time step once for all because it does not change over time. $\overline{\mathbf{K}}$ is a $\left(n_{d} \times n_{d}\right)$ matrix where $n_{d}$ is the number of degrees of freedom of the problem (e.g. $n_{d}=3 \times n_{n}$ where $n_{n}$ is the number of nodes). Having the global matrix, the use of a direct solver such as the LDL factorization is possible. This will further push the simulation closer to the real-time performance as it will allow for pre-computing of the factorization of the matrix before the simulation. Another effect is the significant reduction of the computational load required directly during the simulation.

\section{RESULTS}

To verify the implementation of the FS-FEM performed in SOFA*, a simulation of bending is performed on a cantilever beam with different mesh resolutions (1183, 2210, 4326, 8743 and 12410 elements) and compared to the FEM solution computed using a fine mesh with 22700 elements. The beam is statically loaded by a force of $0.0005 \mathrm{~N}$ applied vertically to the right face of the beam having dimensions $0.178 \times 0.01 \times 0.01 \mathrm{~m}$. The Young's modulus and Poisson's ratio are set to $100 \mathrm{kPa}$ and 0.3 , respectively.

Fig. 2 left shows the convergence of both FEM and FS-FEM for different densities of the mesh. The relative error $\|e\|$ is computed as

$$
\|e\|^{2}=\frac{\int_{\Omega}\left(u^{S F E M}-u_{r e f}^{F E M}\right)^{T}\left(u^{S F E M}-u_{r e f}^{F E M}\right) d \Omega}{\int_{\Omega} u_{r e f}^{F E M^{T}} u_{r e f}^{F E M} d \Omega} .
$$

Fig. 2 right shows the beam deflection obtained from FEM (in green) and FS-FEM (in blue) using the mesh with 2210 elements compared to the reference FEM solution (in red) employing the mesh with 22710 elements.

\footnotetext{
*www.sofa-framework.org
} 

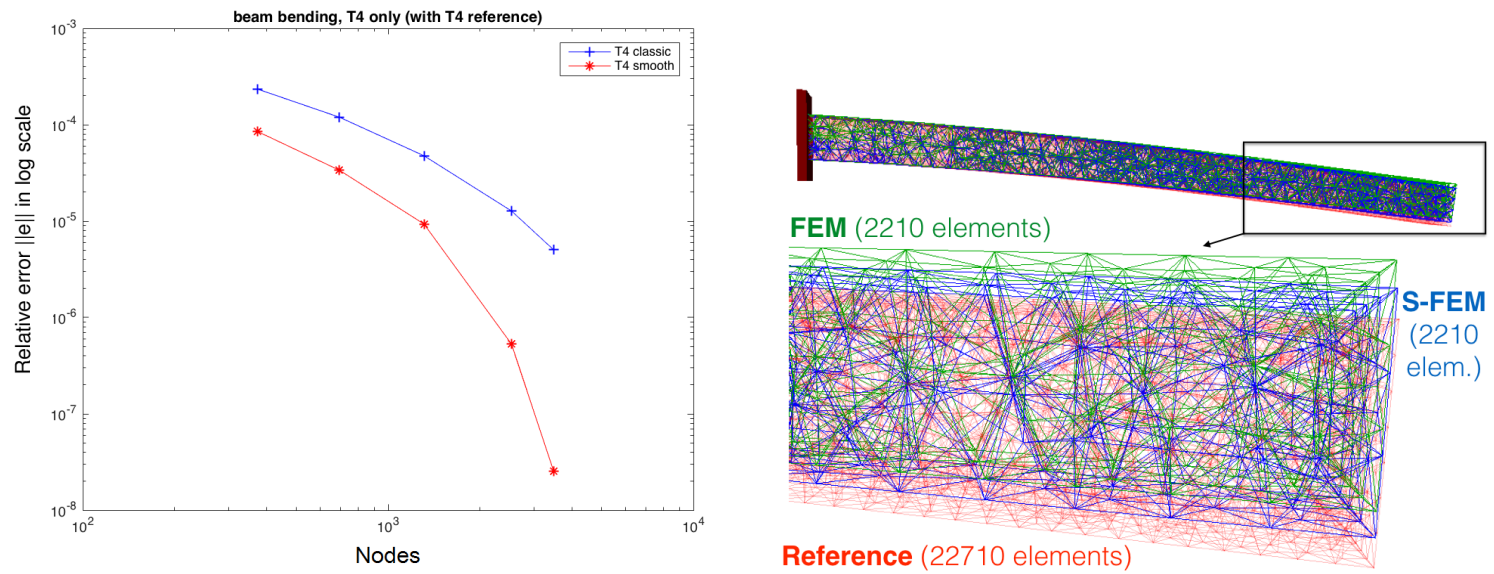

Figure 2. Left : Relative errors of FEM and FS-FEM solution compared to the reference FEM solution (with very fine mesh composed of T4 elements). Right : Zoom-in at the tip of the beams.

It is observed that using the same mesh resolutions, the solutions obtained by FS-FEM are closer to the reference solution when compared to FEM. This confirms our hypothesis that a significantly coarser mesh is sufficient for FS-FEM to achieve the same error as the FEM using finer mesh. This can be in turn exploited in design of a real-time simulation. Further, when using the iterative solver, the rate of convergence is higher when using FS-FEM. It should be emphasized that the amount by which the error decreases is proportional to the difference in number of DOFs required by FEM and FS-FEM.

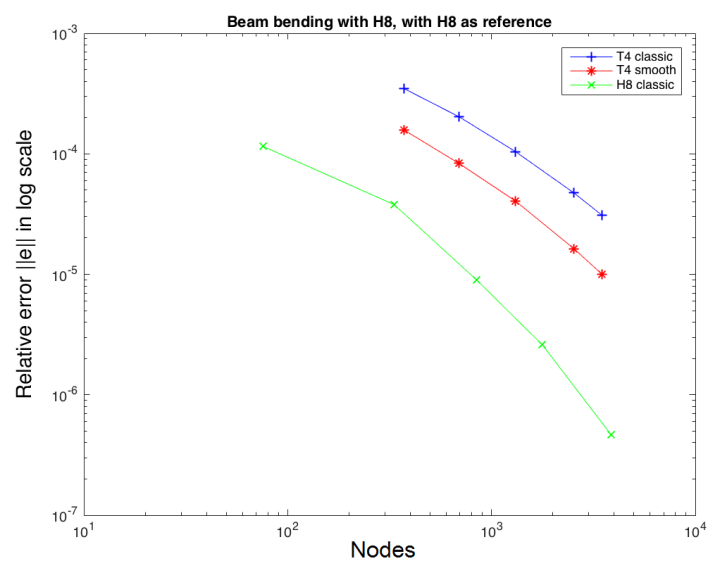

Figure 3. Relative errors in log scale of FEM-T4, FEM-H8 and FS-FEM-T4 solution compared to the reference FEM-H8 solution (with very fine mesh composed of $\mathrm{H} 8$ elements).

We performed the same simulation on the bending beam scenario, but this time employing a FEM with a tetrahedral mesh (FEM-T4), a FEM with an hexahedral mesh (FEM-H8) and a FS-FEM using a tetrahedral mesh (FS-FEM-T4). We run the simulation with 5 different meshes as in the previous experiment. For the tetrahedral meshes we used 373, 693, 1311, 2536 and 3484 nodes, and for the hexahedral ones 76, 333, 845, 1775 and 3852 nodes. The reference solution in this experiment is the result of FEM-H8 having a mesh of 28 000 elements. In Fig. 3 are shown the relative errors computed using equation (9). It is observed that the curve of FS-FEM-T4 is between that of FEM-T4 and FEM-H8. Hence, FS-FEM using T4 elements is closer to a simulation having an hexahedral topology than standard FEM-T4. An hexahedral topology is known for its accuracy but also hexahedral meshes are difficult to generate for complex geometries. Hence, a tetrahedral 
topology is preferred but a high resolution is needed for a high accuracy of the solution. The FS-FEM allows for reducing the mesh resolution while preserving a good accuracy. Overall, the FS-FEM performs better than standard FEM-T4 in a bending scenario.
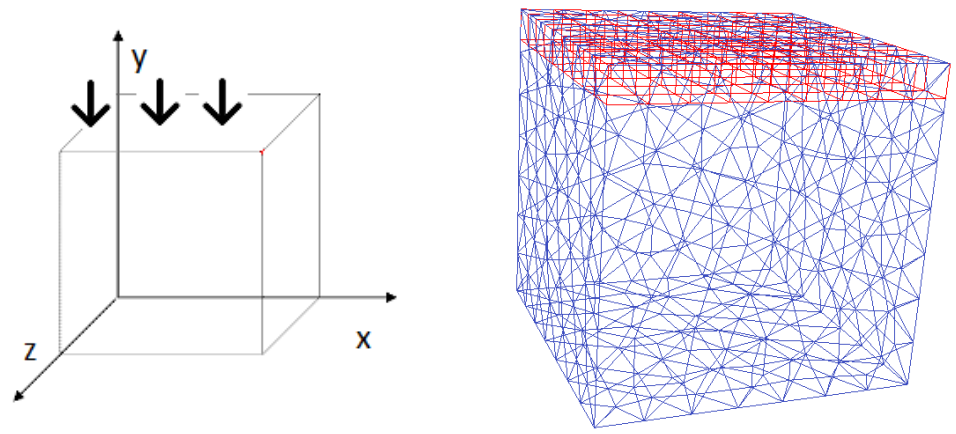

Figure 4. Left : 3D cube under compression. Right: Tetrahedral mesh of the 3D cube and mapped grid in red for the computation of the relative errors.

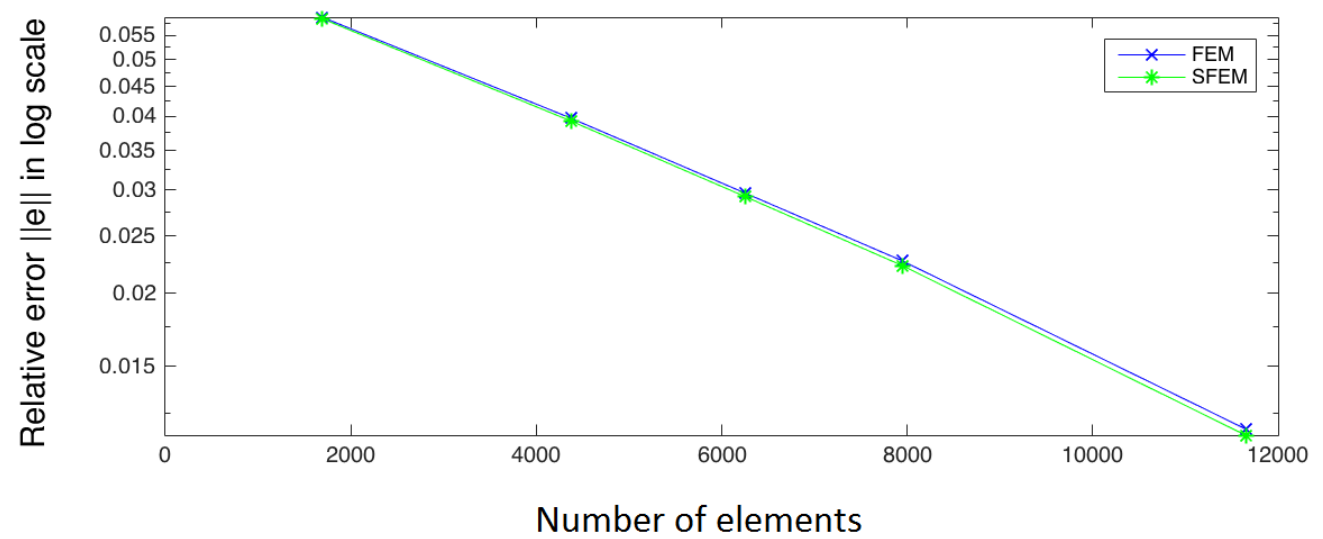

Figure 5. Relative errors in log scale of FEM-T4 and FS-FEM-T4 in cube compression

In the next experiment we analyze the behavior of the FS-FEM in a traction and compression scenario that are common situations in medical contexts. To that purpose we simulated a 3D cube undergoing a uniform load at its upper face with fixed boundary conditions at its lower face as depicted in Fig. 4 left. The Young's modulus and Poisson's ratio of the cube are respectively $2 \mathrm{kPa}$ and 0.45 . We used FEM-T4 and FS-FEM-T4 in meshes having 1423, 2146, 4536, 9553 and 14553 elements. To analyze this compression scenario, we mapped a grid to the upper part of the cube where the deformation is the biggest (cf Fig. 4 right). We computed the relative errors (cf equation (9)) comparing to a reference obtained using a FEM-T4 having 31536 tetrahedral elements. The values are shown in Fig. 5. It is observed that the FS-FEM-T4 has a slightly lower relative error than the FEM-T4 but globally we can say that there is no difference between the two methods in a compression scenario. We find similar results when traction is applied on that cube. Since the strain field is homogeneous over the cube, it was expected to obtain the same result no matter the method used.

Because of its properties, the FS-FEM seems to be a good choice for simulation which requires both high accuracy and good performance. Since this is typically the case of the brain-shift simulation and of the kidney simulation during a partial nephrectomy, we employ the FS-FEM in this two contexts. Fig. 6 shows an image of a human head which was previously used in work of A.Bilger et al. ${ }^{8}$ The anatomy of the brain comes from a 



Figure 6. Brain at rest position and shifted brain simulated with FS-FEM.

generic atlas and its deformations are modeled with the FS-FEM using a tetrahedral mesh. The right image of Fig. 6, shows the result of the simulation, e.g., the completed brain shift predicted by the model. The parameters Young's modulus, Poisson's ratio and mass are set to $3 \mathrm{kPa}, 0.4$ and 0.808 respectively.

Fig. 7 left shows the position of several tumors inside the brain at rest position. Fig. 7 right shows the positions after the deformation due to brain-shift, predicted by a simulation using a linear FEM (blue points), a FS-FEM (red points) and a non-linear FEM (green points). The mesh used for the three methods is a mesh having 7924 tetrahedral elements per hemisphere. Unexpectedly, it is observed that the blue and the red points overlap in most cases. This means that the linear FEM and the FS-FEM do not perform in a significantly different way in this scenario. Furthermore, the red points are in general far from the two other colors. It is very likely that the elements rotate during the deformation and this leads to geometrical non-linearities that are treated better by a non-linear FEM than by the linear FEM or FS-FEM. Overall, the FS-FEM performs qualitatively in the same manner as standard linear FEM in this scenario.
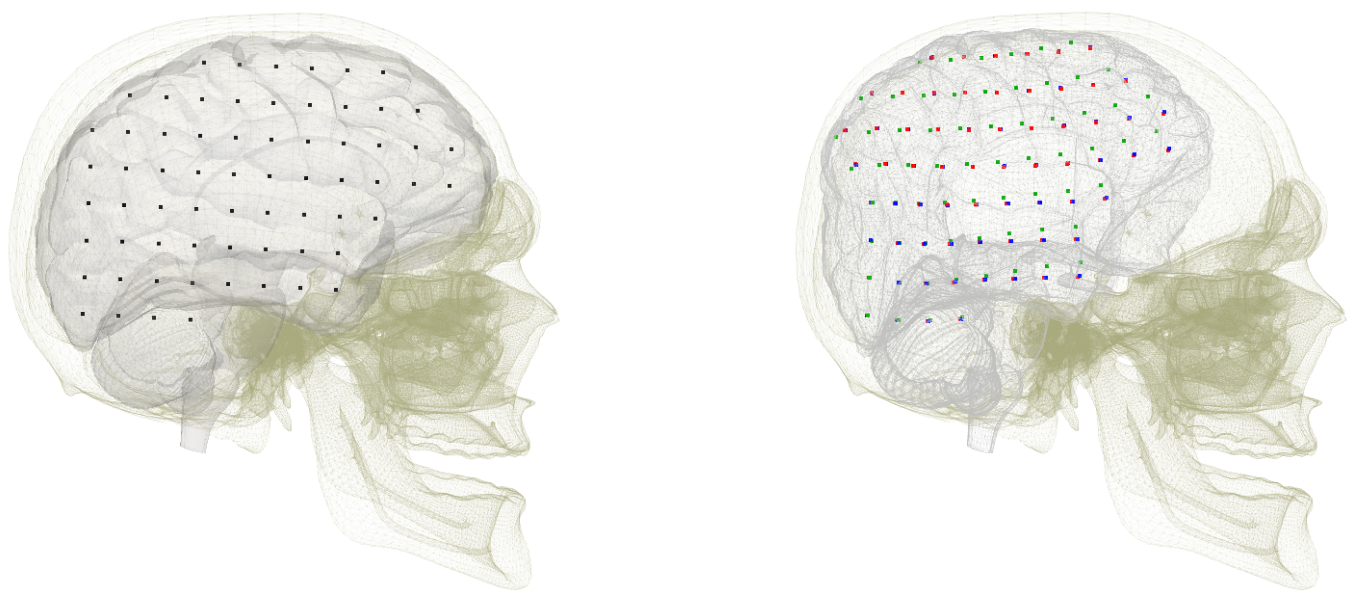

Figure 7. Grid representing tumor positions using a mesh of 7924 elements for linear FEM in blue, FS-FEM in red and non-linear FEM in green after the brain-shift. Left : rest position. Right : position after the deformation due to brain-shift

We now perform a similar simulation and analysis over a kidney under gravity. In Fig. 8, a tetrahedral mesh of a kidney having 727 elements is shown. Its Young's modulus and Poisson's ratio are set to $3 \mathrm{kPa}$ and 0.3 , respectively. We used a direct solver (LDL factorization) in order to be compatible with real-time simulations. 
We want to verify whether the FS-FEM behaves in a similar manner as in the brain. To that purpose, we perform a simulation of the kidney's deformation using the FS-FEM, the linear FEM and a non-linear FEM over a mesh having 727 elements. We placed 8 tumors inside the organ. The position of the tumors after the deformation are shown in Fig. 9 left. The red spheres stand for the FS-FEM, the green ones for the linear FEM and the blue ones for the non-linear FEM. It is observed that all the tumors are very close to each other and as in the brain, in some cases the green spheres are between the red and the blue ones. This means that for the kidney scenario, the FS-FEM performs in a similar manner to the linear FEM, just as in the brain-shit scenario. In Fig. 9 right are shown the results of a simulation comparing the linear FEM and the FS-FEM employing a mesh of 727 elements to a reference solution computed with linear FEM using 3224 elements. The red spheres stand for the FS-FEM, the green ones for the linear FEM and the blue ones for the linear FEM with a very fine mesh. It is observed that the green and the red spheres are overlapping. Hence, it is difficult to draw a conclusion on the most appropriate method for this context. Overall, there is no difference between the FS-FEM and the linear FEM in this scenario.

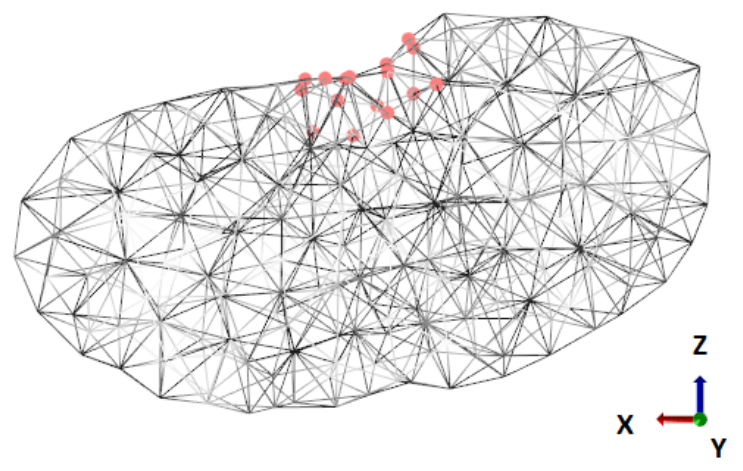

Figure 8. 3D mesh of a kidney with fixed constraints (pink points).

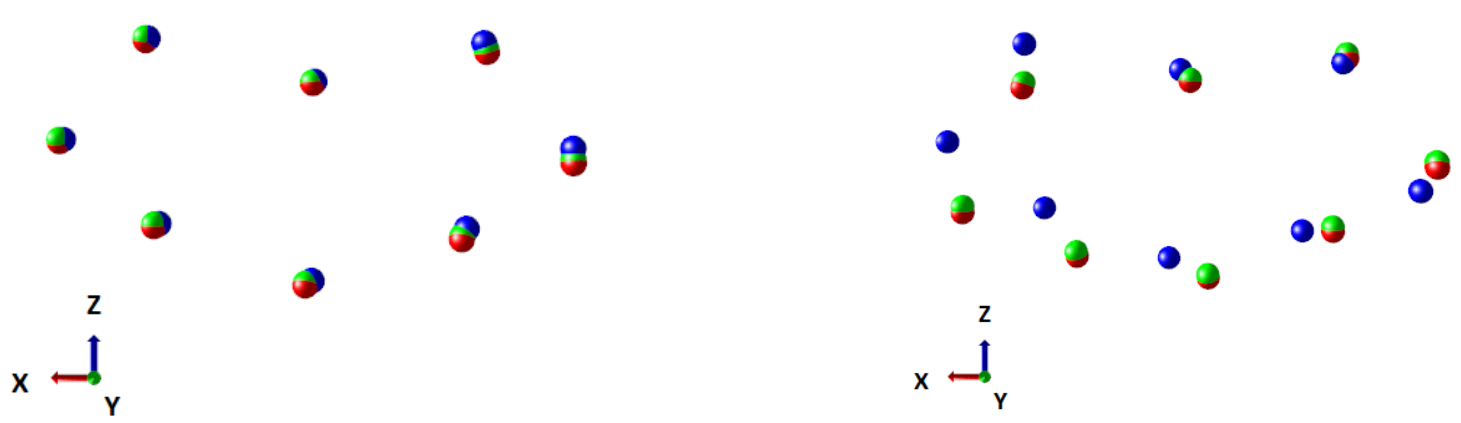

Figure 9. Left : Tumor positions after deforming the kidney under gravity using a FS-FEM having a mesh of 727 elements (red spheres), linear FEM with the same mesh (green spheres) and non-linear FEM with the same mesh (blue spheres). Right : Tumor positions after deforming the kidney under gravity using a FS-FEM having a mesh of 727 elements (red spheres), linear FEM with the same mesh (green spheres) and linear FEM as a reference with a mesh of 3224 elements (blue spheres).

\section{CONCLUSIONS AND PERSPECTIVES}

In a bending scenario, the FS-FEM-T4 is proved to better approximate the displacement solution than the standard FEM-T4. Moreover, the FS-FEM having a tetrahedral mesh is closer to a simulation having an 
hexahedral topology than standard FEM-T4. Hence, the FS-FEM allows for reducing the mesh resolution while preserving a good accuracy of the solution of a bending scenario.

Not surprisingly, our implementation of the FS-FEM presents no advantage on compression and traction scenarios. We have seen that the reason for this is the homogeneous strain field. Indeed, the smoothing has no impact in such cases.

Contrary to our expectations the results on the brain-shift and on the kidney where rather unexpected. We did not find a significant improvement when using the FS-FEM in this contexts.

Hence, further work will concentrate on simulating other organs with different elasticities and shapes such as the liver or the eye in the context of liver tumor removal and retinal surgery. To the best of our knowledge, the co-rotational FEM is the fastest method accounting for geometrical non-linearities and this is a very common situation in medical contexts. As a consequence, our next step is the development of the co-rotational FS-FEM that has not been presented in the literature before.

\section{REFERENCES}

[1] Nguyen-Thoi, T., Liu, G. R., Lam, K. Y., and Zhang, G. Y., "A face-based smoothed finite element method (fs-fem) for 3d linear and geometrically non-linear solid mechanics problems using 4-node tetrahedral elements," International Journal for Numerical Methods in Engineering 78(3), 324-353 (2009).

[2] Duong, M. and Staat, M., "A face-based smoothed finite element method for hyperelastic models and tissue growth," 11 th World Congress on Computational Mechanics, WCCM 2014, 5th European Conference on Computational Mechanics, ECCM 2014 and 6th European Conference on Computational Fluid Dynamics, ECFD 2014, 2657-2668 (2014).

[3] Clatz, O., Delingette, H., Talos, I. F., Golby, A. J., Kikinis, R., Jolesz, F. A., Ayache, N., and Warfield, S. K., "Robust nonrigid registration to capture brain shift from intraoperative mri," IEEE Transactions on Medical Imaging 24, 14171427 (Nov 2005).

[4] Miga, M. I., Paulsen, K. D., Hoopes, P. J., Kennedy, F. E., Hartov, A., and Roberts, D. W., "In vivo quantification of a homogeneous brain deformation model for updating preoperative images during surgery," IEEE Transactions on Biomedical Engineering 47, 266-273 (Feb 2000).

[5] Bucki, M., Lobos, C., and Payan, Y., "Framework for a low-cost intra-operative image-guided neuronavigator including brain shift compensation," in [2007 29th Annual International Conference of the IEEE Engineering in Medicine and Biology Society], 872-875 (Aug 2007).

[6] Nabavi, A., Black, P. M., Gering, D. T., Westin, C. F., Mehta, V., Pergolizzi, R. S., Ferrant, M., Warfield, S. K., Hata, N., Schwartz, R. B., Wells, W. M., Kikinis, R., and Jolesz, F. A., "Serial intraoperative magnetic resonance imaging of brain shift," NEUROSURGERY 48, 787-797 (apr 2001).

[7] Hastreiter, P., Rezk-Salama, C., Soza, G., Bauer, M., Greiner, G., Fahlbusch, R., Ganslandt, O., and Nimsky, C., "Strategies for brain shift evaluation," Medical Image Analysis, 8, 447464 (2004).

[8] Bilger, A., Dequidt, J., Duriez, C., and Cotin, S., [Biomechanical Simulation of Electrode Migration for Deep Brain Stimulation], 339-346, Springer Berlin Heidelberg, Berlin, Heidelberg (2011). 